\title{
ENERGIA METABOLIZÁVEL DE ALIMENTOS UTILIZADOS NA FORMULAÇÃO DE RAÇÕES PARA PAPAGAIOS-VERDADEIROS (Amazona aestiva)
}

\author{
Metabolizable energy of raw feeds utilized in commercial rations \\ for blue-fronted parrot (Amazona aestiva)
}

\author{
Carlos Eduardo do Prado Saad ${ }^{1}$, Walter Motta Ferreira², Flávia Maria de Oliveira Borges ${ }^{3}$, \\ Leonardo Boscoli Lara ${ }^{4}$
}

\begin{abstract}
RESUMO
Para avaliar a energia metabolizável de 16 alimentos comumente utilizados em rações para psitacídeos, foram utilizados 34 papagaios-verdadeiros alojados em gaiolas metabólicas e distribuídos em blocos ao acaso em 17 tratamentos (T1 - ração referência, T2 semente de girassol, T3 - aveia, T4 - gema de ovo, T5 - ovo integral, T6 - clara de ovo, T7 - germe de trigo, T8 - farelo de trigo, T9 - milho moído, T10 - milho gelatinizado, T11- farelo de girassol, T12 - levedura, T13 - polpa cítrica, T14 - mamão, T15 - banana, T16 - farelo de soja, T17 - soja micronizada), durante três períodos de colheita, totalizando seis repetições (102 unidades experimentais). Para avaliação dos alimentos utilizou-se o método de substituição. Foram avaliados os coeficientes de metabolização da energia bruta (aparente $\mathrm{CMA}_{\mathrm{E}}$, verdadeiro $-\mathrm{CMV}_{\mathrm{E}}$, aparente corrigido pelo $\mathrm{N}-\mathrm{CMAn}_{\mathrm{E}}$ e verdadeiro corrigido pelo $\mathrm{N}-\mathrm{CMVn} \mathrm{n}_{\mathrm{E}}$ ) e os valores de energia metabolizável (energia metabolizável aparente - EMA, verdadeira - EMV, aparente corrigida pelo nitrogênio - EMAn e verdadeira corrigida pelo nitrogênio - EMVn) dos alimentos testados. Foram comparados os tratamentos dois a 17. O tratamento um (ração referência) foi utilizado apenas para os cálculos de substituição, não fazendo parte das análises estatísticas. Os coeficientes de metabolização da EB foram comparados pelo teste Scott-Knott, enquanto que para os valores de energia a análise estatística foi descritiva (média e desvio padrão). Os resultados obtidos permitiram concluir que: 1- o valor de energia metabolizável aparente da semente de girassol foi alto e a utilização deste alimento como única fonte de alimento pode, em médio prazo, provocar o surgimento de obesidade nas aves em manutenção; 2- os valores energéticos de alimentos como o milho moído, farelo de trigo, germe de trigo, farelo de soja e soja micronizada para papagaios foram semelhantes àqueles encontrados na literatura para galiformes domésticos, principalmente frango de corte, sugerindo que as extrapolações dos dados de digestibilidade e energia dos alimentos para aves domésticas podem ser utilizadas para papagaios, como forma inicial no cálculo de dietas, até que uma tabela de alimentos para psitacídeos seja definitivamente elaborada.
\end{abstract}

Termos para indexação: Energia metabolizável aparente, energia metabolizável verdadeira, psitacídeos, animais silvestres, aves.

\section{ABSTRACT}

A total of 34 blue fronted parrot were allotted in blocks with 17 treatments (T1 - reference diet, T2 - sunflower seed, T3 - oat, T4 - egg yolk, T5 - integral egg, T6 - egg white, T7 - wheat germen, T8 - wheat bran, T9 - triturated corn, T10 - jellied corn, T11 - sunflower bran, T12 - yeast, T13 - citric pulp, T14 - papaya, T15 - banana, T16 - soy bran, T17 - extruded soy) during three periods, totaling six repetitions (102 experimental units). For feedstuffs evaluation by using a substitution methodology. Were determined the metabolizable coefficients of crude energy (apparent - CMAE, true - CMVE, apparent corrected by N - CMAnE and true corrected by N - CMVnE) and the values of metabolizable energy (apparent - EMA, true - EMV, apparent nitrogen corrected - EMAn and true nitrogen corrected - EMVn) of the tested feedstuffs. All feedstuffs were statistically analyzed. The metabolizable coefficients of EB were compared by the Scott-Knott test , while for the energetics feedstuffs values were analyzed as a descriptive analysis (means and standard deviation). The obtained results allowed to conclude that: 1 - the apparent metabolizable energy of the sunflower seed shown high value and the use of this feed as a only feed source should be used in medium period to avoid the obesity in the birds in maintenance state ; 2 - the energetic feeds as the triturated corn, wheat bran, wheat germen, soybean meal and extruded soy for parrots were similar the those found in the literature for domestic birds, mainly broiler chickens, suggesting that the extrapolation the digestibility energy data of domestic birds mainly broilers should be used for parrots ratios calculations, until that a feedstuffs table for psittacines is set up definitively.

Index terms: Apparent metabolizable energy, true metabolizable energy, psittacines, wild birds.

(Recebido em 4 de novembro de 2005 e aprovado em 27 de março de 2006)

\footnotetext{
'Zootecnista, D.Sc. em Nutrição de Animais Silvestres - AnimalNutri - Rua Dr. Armando Amaral, 122 - Padre Dehon - $37200-000$ - Lavras, MG eduardosaad@animalnutri.com.br; saadzoo@ufla.br

${ }^{2}$ Zootecnista, D.Sc., Professor Adjunto da Escola de Veterinária da Universidade Federal de Minas Gerais/UFMG - Av. Antônio Carlos, 6627 Pampulha - 30123-970 - Belo Horizonte, MG - waltermf@vet.ufmg.br

${ }^{3}$ Médica Veterinária, D.Sc., Professora Adjunta do Departamento de Zootecnia da Universidade Federal de Lavras/UFLA - Cx. P. 3037 - $37200-000-$ Lavras, MG - borgesvet@ufla.br

${ }^{4}$ Médico Veterinário, D.Sc., Nutriara - Rua Jurutau, 1800 - Parque Industrial II - 86703-070 - Arapongas, PR - leoboscoli@gmail.com
} 


\section{INTRODUÇÃO}

Para aves domésticas a formulação de rações envolve o criterioso uso de alimentos e subprodutos combinados de forma a fornecerem quantidades adequadas dos nutrientes requeridos pelas aves. $\mathrm{Na}$ elaboração da maioria das rações para aves silvestres, os valores de nutrientes encontrados nas diversas tabelas de alimentos são extrapolados. Segundo a AAFCO (1998), os valores tabelados dos nutrientes dos alimentos para aves silvestres foram estabelecidos, em sua grande maioria, em experimentos com aves domésticas, podendo não ser aplicáveis para as primeiras. Entretanto, estes valores, embora possam ser utilizados como ponto inicial para formulação de dietas para aves silvestres, podem apresentar diferenças na digestibilidade e nos valores energéticos. Essas diferenças podem ser explicadas devido às características fisiológicas das aves domésticas e silvestres.

Segundo Rodrigues et al. (2002), a energia presente nos alimentos - produto resultante da transformação dos nutrientes durante o metabolismo - é um dos fatores mais importantes na nutrição animal. Os valores que traduzem a utilização da energia pelos animais são aqueles expressos em energia digestível (ED) que significa a energia bruta (EB) do alimento menos a EB das fezes; energia metabolizável (EM) que é igual à EB do alimento menos a EB das fezes, a EB da urina e os gases da digestão; energia líquida (EL) (EM menos o incremento calórico) e energia produtiva (EP) (EL menos energia de manutenção). No caso de aves determina-se diretamente a energia metabolizável, uma vez que urina e fezes são excretadas juntas (BORGES et al., 2003).

O método de avaliação energética mais utilizado é aquele denominado "tradicional", que apresenta como característica primária a utilização de dieta basal administrada a um grupo de aves controle, na qual uma porcentagem desta dieta é substituída pelo ingrediente a ser testado (MATTERSON et al., 1965). Nesse método, o consumo deve ser "ad libitum".

Segundo Rodrigues et al. (2002), nos ensaios biológicos para determinação dos valores energéticos dos alimentos, o balanço de nitrogênio pode ser positivo ou negativo. A retenção de nitrogênio pode ser afetada por vários fatores, dentre os quais se incluem o consumo e a composição do alimento fornecido. O nitrogênio dietético retido no corpo, se catabolisado, é excretado na forma de compostos contendo energia, como o ácido úrico. Assim, é comum a correção dos valores de energia metabolizável aparente (EMA) para balanço de nitrogênio igual a zero, podendo-se determinar a energia metabolizável aparente corrigida pelo nitrogênio (EMAn) e a energia metabolizável verdadeira corrigida pelo nitrogênio (EMVn).

Assim, visou-se com este trabalho determinar os coeficientes de digestibilidade da energia bruta e os valores de energia metabolizável aparente, aparente corrigida pelo nitrogênio, verdadeira e verdadeira corrigida pelo nitrogênio de vários alimentos e matérias-primas utilizadas na composição de rações para papagaios.

\section{MATERIAL E MÉTODOS}

O experimento foi realizado no Departamento de Zootecnia da Universidade Federal de Minas Gerais, onde foram utilizados 34 papagaios-verdadeiros (Amazona aestiva), individualizados em gaiolas metabólicas, distribuídos em blocos ao acaso, em 17 tratamentos (T1 ração referência, T2 - semente de girassol, T3 - aveia, T4 gema de ovo, T5 - ovo integral, T6 - clara de ovo, T7 germe de trigo, T8 - farelo de trigo, T9 - milho moído, T10 milho gelatinizado,T11- farelo de girassol, T12 - levedura, T13 - polpa cítrica, T14 - mamão, T15 - banana, T16 - farelo de soja e T17 - soja micronizada), durante três períodos de colheita - onde cada período constituía um bloco totalizando seis repetições (102 unidades experimentais).

Para avaliação dos alimentos utilizou-se o método de substituição descrita por Matterson et al. (1965), sendo o T1 (ração referência) uma ração extrusada para psitacídeos. As dietas foram compostas por $29,76 \%$ (base da matéria natural) dos alimentos testados (T3-T17), 0,24\% de premix vitamínico mineral, para que todas as rações apresentassem o mesmo teor de minerais e vitaminas, e $70 \%$ da ração referência (T1). Posteriormente todas as rações foram extrusadas, igualando a forma de apresentação dos tratamentos, com exceção à semente de girassol (T2) que foi oferecida "in natura". Porém, como os papagaios não consomem as cascas das sementes, foi estimada uma relação de $65,83 \%$ de semente e $34,17 \%$ de cascas a partir do descascamento manual de 500 gramas de semente, para a avaliação da semente de girassol.

As rações foram fornecidas à vontade durante 12 dias (sete dias de adaptação e cinco de colheita de dados) em cada um dos três períodos e registraram-se as quantidades de ração-teste ingeridas por unidade experimental, tomando-se o cuidado de separar e pesar as sobras retidas nas bandejas de excreta.

Para a avaliação dos coeficientes de digestibilidade da energia bruta e valores de energia metabolizável, procedeu-se a colheita total de excreta. Ao final do terceiro período experimental todas as aves foram colocadas em jejum durante 72 horas para a obtenção do fator de correção 
da energia metabolizável verdadeira. As aves em jejum receberam uma solução de água e glicose (cinco gramas de glicose/ave/dia), conforme recomendado por Macnab \& Blair (1988). Esta fonte energética mantém o metabolismo basal das aves minimizando a interferência do não fornecimento de alimentos nas perdas endógenas.

Os parâmetros avaliados foram os coeficientes de metabolização da energia bruta (aparente - $\mathrm{CMA}_{\mathrm{E}}$, verdadeiro - $\mathrm{CMV}_{\mathrm{E}}$, aparente corrigido pelo $\mathrm{N}-\mathrm{CMAn}_{\mathrm{E}} \mathrm{e}$ verdadeiro corrigido pelo $\mathrm{N}-\mathrm{CMVn_{ \textrm {E } }}$ ) e os valores de energia metabolizável (aparente -EMA, verdadeira - EMV, aparente corrigida pelo nitrogênio - EMAn e verdadeira corrigida pelo nitrogênio - EMVn).

As análises químicas das amostras das rações teste (Tabela 1), dos alimentos puros (Tabela 2) e das excretas foram realizadas no Laboratório de Nutrição Animal da Escola de Veterinária da UFMG e no Laboratório de Nutrição do Instituto Mineiro de Agropecuária, segundo a metodologia da AOAC (CUNNIFF, 1995). A determinação da energia Bruta (EB) foi realizada utilizando-se calorímetro adiabático PARR e as médias $\operatorname{dos} \mathrm{CMA}_{\mathrm{E}}, \mathrm{CMAn}_{\mathrm{E}}, \mathrm{CMV}_{\mathrm{E}}$ e CMVn $\mathrm{E}_{\mathrm{E}}$ foram comparadas pelo teste Scott-Knott.

\section{RESULTADOS E DISCUSSÃO}

Os valores de energia metabolizável aparente (EMA), aparente corrigida pelo nitrogênio (EMAn), verdadeira (EMV) e verdadeira corrigida pelo nitrogênio (EMVn), em kcal/kg de MS, dos alimentos avaliados encontram-se na Tabela 3.

Os teores de EMA e EMAn dos alimentos apresentaram menores valores energéticos que as EMV e EMVn, sendo que a EMV foi, em média, 3,01\% maior que a EMA e a EMVn 6,31 \% maior que a EMAn. Estes resultados mostram a influência das perdas endógenas e metabólicas nos valores de energia metabolizável aparente. Estas perdas são consideradas como energia perdida nas excretas e conseqüientemente subestimam os valores de EMA e EMAn.

Quase todos os resultados de EMA e EMV foram mais altos que os valores de EMAn e EMVn, o que demonstra que as aves ainda se encontravam em balanço positivo de nitrogênio. As exceções foram os valores de EM do farelo de trigo, polpa cítrica e banana, que apresentaram valores de EMA e EMV ligeiramente inferiores ao de EMAn e EMVn.

Tabela 1 - Análises químicas (\%) e valores de energia bruta $(\mathrm{kcal} / \mathrm{g})$ das rações experimentais ${ }^{1}$.

\begin{tabular}{lrrrrrrrr}
\hline Rações & $\mathrm{MS}^{2}$ & $\mathrm{MO}^{2}$ & $\mathrm{~EB}^{2}$ & $\mathrm{~PB}^{2}$ & $\mathrm{Cz}^{2}$ & $\mathrm{FB}^{2}$ & $\mathrm{Ca}^{2}$ & $\mathrm{P}^{2}$ \\
\hline Ração referência & 91,73 & 86,98 & 4560 & 18,01 & 5,18 & 2,26 & 0,99 & 0,87 \\
Ração Aveia & 90,97 & 86,76 & 4647 & 17,02 & 4,63 & 2,31 & 0,78 & 0,82 \\
Ração Gema de ovo & 93,64 & 89,07 & 5032 & 22,27 & 4,88 & 2,90 & 0,83 & 1,07 \\
Ração Ovo Integral & 91,36 & 86,85 & 4957 & 27,12 & 4,94 & 2,04 & 0,76 & 0,43 \\
Ração Clara de ovo & 93,37 & 88,00 & 4817 & 38,39 & 5,76 & 2,22 & 0,72 & 0,55 \\
Ração Germe de trigo & 92,89 & 88,13 & 4657 & 21,77 & 5,13 & 2,33 & 0,73 & 0,92 \\
Ração Farelo de trigo & 93,26 & 88,33 & 4558 & 16,99 & 5,29 & 5,03 & 0,77 & 1,12 \\
Ração Milho moído & 90,71 & 86,90 & 4549 & 15,36 & 4,19 & 2,61 & 1,23 & 0,80 \\
Ração Milho gelatinizado & 91,36 & 87,65 & 4527 & 15,67 & 4,06 & 1,94 & 0,71 & 0,88 \\
Ração Farelo de girassol & 91,43 & 86,06 & 4707 & 20,94 & 5,88 & 10,48 & 0,82 & 0,87 \\
Ração Levedura de cerveja & 91,89 & 86,69 & 4635 & 25,44 & 5,66 & 2,07 & 0,81 & 0,93 \\
Ração Polpa cítrica & 92,41 & 87,71 & 4510 & 15,95 & 5,09 & 3,96 & 1,04 & 0,67 \\
Ração Mamão desidratado & 93,12 & 87,82 & 4414 & 13,02 & 5,69 & 1,59 & 0,72 & 0,88 \\
Ração Banana desidratada & 91,93 & 87,60 & 4493 & 13,45 & 4,71 & 1,85 & 0,73 & 0,69 \\
Ração Farelo de soja & 90,85 & 86,05 & 4692 & 26,43 & 5,28 & 2,85 & 0,70 & 0,88 \\
Ração Soja micronizada & 92,89 & 87,81 & 4967 & 24,85 & 5,47 & 2,20 & 0,78 & 0,86 \\
\hline
\end{tabular}

Obs: Todas as rações passaram por um processo de extrusão.

1- Dados expressos com base na matéria seca.

2- $\mathrm{MS}$ =matéria seca, $\mathrm{MO}=$ matéria orgânica, $\mathrm{EB}=$ energia bruta, $\mathrm{PB}=$ proteína bruta, $\mathrm{Cz}=$ cinzas, $\mathrm{FB}=$ fibra bruta, $\mathrm{Ca}=\mathrm{Cálcio} \mathrm{e}$ $\mathrm{P}=$ Fósforo. 
Tabela 2 - Análises químicas (\%) e valores de energia bruta (kcal/kg) dos alimentos ${ }^{1}$.

\begin{tabular}{lcccccccccc}
\hline Alimentos & $\mathrm{MS}^{2}$ & $\mathrm{MO}^{2}$ & $\mathrm{~EB}^{2}$ & $\mathrm{~PB}^{2}$ & $\mathrm{FB}^{2}$ & $\mathrm{EE}^{2}$ & $\mathrm{Cz}^{2}$ & $\mathrm{ENN}^{2}$ & $\mathrm{Ca}^{2}$ & $\mathrm{P}^{2}$ \\
\hline Semente de Girassol & 95,90 & 93,04 & 7643 & 24,17 & 2,86 & 57,80 & 2,98 & 12,19 & 0,06 & 1,05 \\
Aveia & 89,00 & 86,96 & 4873 & 13,95 & 1,68 & 9,12 & 2,29 & 72,96 & 0,09 & 0,43 \\
Gema de ovo & 97,22 & 91,25 & 6469 & 38,69 & $\mathrm{ND}$ & 46,87 & 6,14 & 8,31 & 0,31 & 0,55 \\
Ovo Integral & 95,65 & 91,25 & 6228 & 51,84 & $\mathrm{ND}$ & 35,41 & 4,60 & 8,16 & 0,35 & 1,09 \\
Clara de ovo & 93,83 & 86,36 & 5081 & 88,72 & $\mathrm{ND}$ & 0,66 & 7,96 & 2,66 & 0,06 & 0,21 \\
Germe de trigo & 88,65 & 83,87 & 4923 & 29,46 & 2,42 & 9,18 & 5,39 & 53,56 & 0,06 & 0,73 \\
Farelo de trigo & 88,92 & 84,08 & 4781 & 15,69 & 9,35 & 5,17 & 5,44 & 64,36 & 0,12 & 0,65 \\
Milho moído & 87,47 & 86,47 & 4495 & 9,01 & 1,19 & 5,07 & 1,14 & 83,60 & 0,02 & 0,46 \\
Milho gelatinizado & 90,43 & 89,24 & 4512 & 8,84 & 1,46 & 2,38 & 1,31 & 86,01 & 0,02 & 0,31 \\
Farelo de girassol & 91,00 & 87,13 & 4743 & 27,24 & 27,35 & 3,44 & 4,25 & 37,72 & 0,24 & 0,58 \\
Levedura & 90,13 & 83,55 & 4688 & 44,82 & $\mathrm{ND}$ & 0,68 & 7,30 & 47,20 & 0,10 & 1,82 \\
Polpa cítrica & 88,81 & 84,29 & 4389 & 11,66 & 9,43 & 3,36 & 5,09 & 70,46 & 1,08 & 0,28 \\
Mamão desidratado & 94,24 & 92,36 & 4150 & 1,95 & $\mathrm{ND}$ & 1,03 & 1,99 & 95,04 & 0,06 & 0,27 \\
Banana desidratada & 91,73 & 87,48 & 4163 & 5,45 & $\mathrm{ND}$ & 0,32 & 4,63 & 89,60 & 0,02 & 0,20 \\
Farelo de soja & 87,90 & 82,68 & 4820 & 50,41 & 4,81 & 3,85 & 5,94 & 34,99 & 0,28 & 0,77 \\
Soja micronizada & 94,89 & 90,56 & 5815 & 41,92 & 0,49 & 25,19 & 4,56 & 27,85 & 0,13 & 0,59 \\
\hline
\end{tabular}

1- Dados expressos na base da matéria seca.

2- $\mathrm{MS}=$ matéria seca, $\mathrm{MO}=$ matéria orgânica, $\mathrm{EB}=$ energia bruta, $\mathrm{PB}=$ proteína bruta, $\mathrm{FB}=$ fibra bruta, $\mathrm{EE}=$ extrato etéreo, $\mathrm{Cz}=$ cinzas, $\mathrm{ENN}=$ Extrativo não nitrogenado, $\mathrm{Ca}=$ Cálcio, $\mathrm{P}=$ Fósforo e ND = Não detectado pelos limites da técnica.

3- Valores na semente de girassol descorticada manualmente.

Tabela 3 - Médias e desvios padrão da energia metabolizável aparente (EMA), aparente corrigida pelo nitrogênio (EMAn), verdadeira (EMV) e verdadeira corrigida pelo nitrogênio (EMVn), em kcal $/ \mathrm{kg}$ de MS dos alimentos avaliados.

\begin{tabular}{lcccccccccccc}
\hline Alimentos & \multicolumn{3}{c}{ EMA } & \multicolumn{3}{c}{ EMAn } & \multicolumn{3}{c}{ EMV } & \multicolumn{3}{c}{ EMVn } \\
\hline Semente de Girassol & 7049,6 & \pm & 87,3 & 6884,1 & \pm & 65,8 & 7189,7 & \pm & 114,9 & 6942,0 & \pm & 145,0 \\
Aveia & 3846,4 & \pm & 164,4 & 3765,9 & \pm & 192,6 & 4006,5 & \pm & 164,6 & 3886,6 & \pm & 225,9 \\
Gema de ovo & 5469,2 & \pm & 169,9 & 5298,6 & \pm & 173,7 & 5722,0 & \pm & 169,7 & 5452,9 & \pm & 205,7 \\
Ovo Integral & 5040,0 & \pm & 116,8 & 4781,1 & \pm & 152,0 & 5186,0 & \pm & 127,3 & 4892,1 & \pm & 183,4 \\
Clara de ovo & 3433,1 & \pm & 162,3 & 3124,3 & \pm & 184,7 & 3518,9 & \pm & 117,9 & 3211,6 & \pm & 138,3 \\
Germe de trigo & 3043,8 & \pm & 105,0 & 2930,2 & \pm & 102,0 & 3106,7 & \pm & 96,7 & 3011,0 & \pm & 136,1 \\
Farelo de trigo & 2021,1 & \pm & 321,0 & 2025,4 & \pm & 257,9 & 2036,7 & \pm & 275,0 & 2086,5 & \pm & 190,2 \\
Milho moído & 3634,8 & \pm & 148,7 & 3647,4 & \pm & 121,5 & 3800,3 & \pm & 87,1 & 3771,3 & \pm & 211,9 \\
Milho gelatinizado & 4059,7 & \pm & 236,8 & 3998,5 & \pm & 201,3 & 4147,5 & \pm & 212,5 & 4088,5 & \pm & 159,7 \\
Farelo de girassol & 1900,0 & \pm & 435,8 & 1773,2 & \pm & 461,2 & 1922,5 & \pm & 425,5 & 1835,9 & \pm & 443,2 \\
Levedura de Cerveja & 2893,8 & \pm & 280,8 & 2700,9 & \pm & 274,9 & 2945,4 & \pm & 251,4 & 2776,1 & \pm & 220,6 \\
Polpa cítrica & 1025,5 & \pm & 200,4 & 1069,5 & \pm & 171,8 & 1059,5 & \pm & 181,2 & 1138,3 & \pm & 138,7 \\
Mamão Desidratado & 3306,2 & \pm & 245,8 & 3286,1 & \pm & 140,0 & 3414,9 & \pm & 188,8 & 3382,5 & \pm & 83,5 \\
Banana Desidratada & 3284,2 & \pm & 204,0 & 3297,9 & \pm & 229,6 & 3420,7 & \pm & 213,8 & 3427,3 & \pm & 249,7 \\
Farelo de soja & 2792,2 & \pm & 223,5 & 2634,2 & \pm & 270,1 & 2921,6 & \pm & 255,4 & 2742,9 & \pm & 361,3 \\
Soja micronizada & 4272,8 & \pm & 163,6 & 4081,4 & \pm & 103,5 & 4394,2 & \pm & 138,1 & 4182,8 & \pm & 174,2 \\
\hline Média & 3567,0 & \pm & 1457,4 & 3456,2 & \pm & 1413,8 & 3674,6 & \pm & 1502,6 & 3550,5 & \pm & 1423,1 \\
\hline
\end{tabular}


Os alimentos que apresentaram maiores valores de energia metabolizável foram a semente de girassol, seguida pela gema de ovo e pelo ovo integral, enquanto que os menores teores foram encontrados na polpa cítrica, nos farelos de girassol e trigo. Estes resultados eram esperados, visto que os alimentos com maiores teores energéticos são aqueles que apresentaram os maiores teores de extrato etéreo (Tabela 2), enquanto que os alimentos com menor energia metabolizável foram aqueles com maior teor de carboidratos estruturais.

Os valores de energia metabolizável aparente da semente de girassol foram mais altos que os encontrados na literatura para aves similares. Wolf (2002) cita valor de $6000 \mathrm{kcal} / \mathrm{kg}$ de EM para a semente descorticada, enquanto que Mark-Hagen (2001), trabalhando com Cacatuas, encontrou valores de $6207 \pm 282 \mathrm{kcal} / \mathrm{kg}$, para as sementes descorticadas, quando oferecidas "ad libitum" e $6094 \pm$ $86 \mathrm{kcal} / \mathrm{kg}$ quando determinado pelo método da alimentação forçada. Estas diferenças podem ser devidas à origem das sementes, uma vez que no experimento de Mark-Hagen (2001) o valor encontrado de energia bruta para as sementes foi de $7009 \mathrm{kcal} / \mathrm{kg}$, enquanto que neste experimento o valor foi mais alto, de $7643 \mathrm{kcal} / \mathrm{kg}$. O conteúdo energético alto da semente de girassol aliado à sua alta palatabilidade pode provocar o surgimento de obesidade em papagaios em manutenção, portanto, não deve ser usada como única fonte de alimentação neste caso.

O valor de energia metabolizável aparente encontrado para a aveia foi de $3846,4 \mathrm{kcal} / \mathrm{kg}$, aproximandose do valor de EM deste alimento para papagaios, citado por Wolf (2002), de $4020 \mathrm{kcal} / \mathrm{kg}$.

Não foi encontrada na literatura consultada, nenhuma referência aos valores de energia metabolizável da gema, da clara ou do ovo integral para psitacídeos. Os valores de EMA encontrados neste experimento foram de 5469,$2 ; 5040,0$ e $3433,1 \mathrm{kcal} / \mathrm{kg}$, para os três alimentos, respectivamente. $\mathrm{O}$ maior teor de EM encontrado para a gema de ovo se deve, provavelmente, ao maior teor de óleo deste alimento. Dierenfeld \& Wendy (1996) citam o valor de $5830 \mathrm{kcal} / \mathrm{kg}$ de EM para o ovo cozido sem casca, entretanto este valor foi obtido em tabela de nutrição de humanos.

A EMA do milho gelatinizado $(4059,7 \mathrm{kcal} / \mathrm{kg})$ foi $11,5 \%$ mais alta que a do milho moído $(3634,8 \mathrm{kcal} / \mathrm{kg})$ embora ambos tenham apresentado valores de EB bem semelhantes (4495 e $4512 \mathrm{Kcal} / \mathrm{kg}$, respectivamente). É possível que o método de processamento (gelatinização) seja responsável por este incremento na energia metabolizável.
O mesmo ocorreu com a soja micronizada, que apresentou um valor de EMA 53\% superior ao do farelo de soja (4272,8 e 2792,2 $\mathrm{kcal} / \mathrm{kg}$, respectivamente), entretanto, neste caso, os valores de energia bruta dos dois alimentos foram bem distintos (5815 e $4820 \mathrm{kcal} / \mathrm{kg}$ ). Novamente, pode-se inferir que os resultados se devam, principalmente, aos teores de fibra $(0,49$ e $4,81 \%$, respectivamente) que atuam diminuindo os níveis de energia, conjugados aos teores de extrato etéreo $(3,85$ e $25,19 \%$ para o farelo e para a soja micronizada, respectivamente) que, normalmente, apresentam alta digestibilidade e altos valores de energia.

$O$ resultado de EMA encontrado neste experimento para a soja micronizada $(4272,8 \mathrm{kcal} / \mathrm{kg})$ foi semelhante ao encontrado por Rodrigues et al. (2002) em experimento com ração farelada para pintos $(4260 \mathrm{kcal} /$ $\mathrm{kg}$ ), como este alimento já foi micronizado, teoricamente sofre pouca influência da extrusão com relação aos coeficientes de digestibilidade. Já com relação aos farelos, o referido autor avaliou farelos de diferentes procedências e o mais alto valor encontrado foi $2633 \mathrm{kcal} / \mathrm{kg}$, inferior ao resultado de EMA do farelo de soja obtido em ração extrusada para papagaios neste experimento, que foi $2792,2 \mathrm{kcal} / \mathrm{kg}$. Neste caso as diferenças podem ser devidas à apresentação das rações, farelada e extrusadas, respectivamente, uma vez que a extrusão pode alterar o valor da EMA dos farelos.

Os valores de EMA para o mamão e a banana foram muito similares (3306,2 e 3284,2 kcal/kg, respectivamente), e os valores encontrados da energia bruta também foram semelhantes (4150 e $4163 \mathrm{kcal} / \mathrm{kg}$ ). O valor de EMA para o mamão encontrado neste experimento foi semelhante ao obtido por Dierenfeld \& Wendy (1996), de $3310 \mathrm{kcal} / \mathrm{kg}$, entretanto, o valor citado por esses autores para a banana, de $3540 \mathrm{kcal} / \mathrm{kg}$ foi mais elevado que o encontrado neste experimento, de $3284,2 \mathrm{kcal} / \mathrm{kg}$.

Os coeficientes de metabolização aparente $\left(\mathrm{CMA}_{\mathbf{E}}\right)$, aparente corrigida pelo nitrogênio $\left(\mathrm{CMAn}_{\mathrm{E}}\right)$, verdadeiro $\left(\mathrm{CMV}_{\mathbf{E}}\right)$ e verdadeiro corrigido pelo nitrogênio $\left(\mathrm{CMVn}_{\mathbf{E}}\right)$, da energia bruta (\%) dos alimentos avaliados encontramse na Tabela 4. Estes dados são apresentados para mostrar que, embora muitos alimentos tenham apresentado valores de EM bastante distintos, como, por exemplo, a semente de Girassol (7049,6 kcal/kg) e o milho gelatinizado (4059,7 $\mathrm{kcal} / \mathrm{kg}$ ), seus coeficientes de metabolização da EB foram semelhantes $(92,23$ e $89,97 \%$, respectivamente) estatisticamente. Estes dois alimentos apresentaram o mais alto coeficiente de metabolização $(\mathrm{P}<0,05)$ da energia, para $\mathrm{CMA}_{\mathbf{E}}, \mathrm{CMAn}_{\mathbf{E}}, \mathrm{CMV}_{\mathbf{E}}$ e CMVn $\mathbf{E}^{\cdot}$ 
Tabela 4 - Coeficientes de metabolização aparente $\left(\mathrm{CMA}_{\mathrm{E}}\right)$, aparente corrigida pelo nitrogênio $\left(\mathrm{CMAn} \mathrm{E}_{\mathrm{E}}\right)$, verdadeiro $\left(\mathrm{CMV}_{\mathrm{E}}\right)$ e verdadeiro corrigida pelo nitrogênio $\left(\mathrm{CMVn}_{\mathrm{E}}\right)$, da energia bruta $(\%)$ dos alimentos avaliados.

\begin{tabular}{|c|c|c|c|c|}
\hline Alimentos & $\mathrm{CMA}_{\mathrm{E}}$ & $\mathrm{CMAn}_{\mathrm{E}}$ & $\mathrm{CMV}_{\mathrm{E}}$ & $\mathrm{CMVn}_{\mathrm{E}}$ \\
\hline Semente de Girassol & $92,23^{1} \mathrm{a}$ & $90,07 \mathrm{a}$ & $94,06 \mathrm{a}$ & $90,82 \mathrm{a}$ \\
\hline Aveia & $78,93 \mathrm{~b}$ & $77,28 \mathrm{~b}$ & $82,22 \mathrm{~b}$ & $79,75 \mathrm{~b}$ \\
\hline Gema de ovo & $84,54 \mathrm{~b}$ & $81,90 \mathrm{~b}$ & $88,45 \mathrm{a}$ & $84,29 \mathrm{~b}$ \\
\hline Ovo Integral & $80,93 \mathrm{~b}$ & $76,77 \mathrm{~b}$ & $83,27 b$ & $78,55 \mathrm{~b}$ \\
\hline Clara de ovo & $67,56 \mathrm{c}$ & $61,48 \mathrm{~d}$ & $69,25 \mathrm{~d}$ & $63,20 \mathrm{~d}$ \\
\hline Germe de trigo & $61,83 \mathrm{c}$ & $59,52 \mathrm{~d}$ & $63,10 \mathrm{e}$ & $61,16 \mathrm{~d}$ \\
\hline Farelo de trigo & $42,27 \mathrm{~d}$ & $42,35 \mathrm{e}$ & $42,60 \mathrm{f}$ & $43,64 \mathrm{e}$ \\
\hline Milho moído & $80,86 \mathrm{~b}$ & $81,14 b$ & $84,54 \mathrm{~b}$ & $83,90 \mathrm{~b}$ \\
\hline Milho gelatinizado & $89,97 \mathrm{a}$ & $88,61 \mathrm{a}$ & $91,91 \mathrm{a}$ & $90,61 \mathrm{a}$ \\
\hline Farelo de girassol & $40,06 \mathrm{~d}$ & $37,38 \mathrm{e}$ & $40,53 \mathrm{f}$ & $38,70 \mathrm{e}$ \\
\hline Levedura de Cerveja & $61,72 \mathrm{c}$ & $57,60 \mathrm{~d}$ & $62,82 \mathrm{e}$ & $59,21 \mathrm{~d}$ \\
\hline Polpa cítrica & $23,36 \mathrm{e}$ & $24,36 \mathrm{f}$ & $24,14 \mathrm{~g}$ & $25,93 \mathrm{f}$ \\
\hline Mamão Desidratado & $79,66 \mathrm{~b}$ & $79,18 \mathrm{~b}$ & $82,28 \mathrm{~b}$ & $81,50 \mathrm{~b}$ \\
\hline Banana Desidratada & $78,89 \mathrm{~b}$ & $79,22 \mathrm{~b}$ & $82,17 \mathrm{~b}$ & $82,85 \mathrm{~b}$ \\
\hline Farelo de soja & $57,93 \mathrm{c}$ & $54,65 \mathrm{~d}$ & $60,62 \mathrm{e}$ & $56,91 \mathrm{~d}$ \\
\hline Soja micronizada & $73,48 \mathrm{~b}$ & $70,19 \mathrm{c}$ & $75,57 \mathrm{c}$ & $71,93 \mathrm{c}$ \\
\hline $\mathrm{CV}(\%)$ & 6,86 & 6,51 & 6,39 & 6,27 \\
\hline
\end{tabular}

Valores em uma mesma coluna, seguidos de letras distintas, diferem pelo teste de Scott-Knott $(\mathrm{P}<0,05)$.

Do mesmo modo, os valores de $\mathrm{CMA}_{\mathrm{E}}$ da aveia, da gema de ovo, do ovo integral, do milho moído, do mamão, da banana e da soja micronizada $(78,93,84,54,80,93,80,86$, $79,66,78,89$ e 73,48\%, respectivamente) não diferiram entre si $(\mathrm{P}>0,05)$, mostrando-se inferiores apenas aos valores de $\mathrm{CMA}_{\mathrm{E}}$ da semente de girassol e do milho gelatinizado. $\mathrm{O}$ mesmo ocorreu para CMAn $\mathbf{E}_{\mathbf{E}} \mathrm{CMV}_{\mathbf{E}}$ e $\mathrm{CMVn}_{\mathbf{E}}$, com exceção ao valor encontrado para a gema de ovo $(88,45 \%)$, que apresentou valores superiores aos encontrados nos alimentos aveia, ovo integral, milho moído, mamão, banana e soja micronizada.

Os CMAn CMV $_{\mathbf{E}}$ e CMVn para a semente de girassol e para o milho gelatinizado foram semelhantes ( $\mathrm{P}>0,05)$. Já a soja micronizada apresentou os menores valores $(\mathrm{P}>0,05)$ dentre os alimentos testados.

Valores intermediários e estatisticamente semelhantes $(\mathrm{P}>0,05)$ de $\mathrm{CMA}_{\mathrm{E}}$ foram encontrados para a clara de ovo, o germe de trigo, a levedura de cerveja e o farelo de soja, sendo que estes alimentos apresentaram valores estatisticamente maiores $(67,56 ; 61,83 ; 61,72$ e 57,93\%, respectivamente) que aqueles dos farelos de trigo e girassol (42,27 e 40,06\%, respectivamente). Estes dois últimos alimentos apresentaram baixos valores significativamente semelhantes de $\mathrm{CMA}_{\mathbf{E}} \mathrm{CMAn}_{\mathbf{E}}, \mathrm{CMV}_{\mathbf{E}}$ e CMVn $\mathbf{E}_{\mathbf{E}}$.
Finalmente, a polpa cítrica apresentou o menor valor

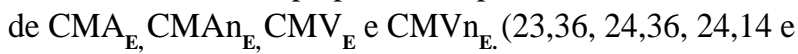
$25,93 \%$, respectivamente), diferindo estatisticamente de todos os outros alimentos.

\section{CONCLUSÕES}

Os resultados obtidos neste estudo permitem as seguintes conclusões:

O valor de energia metabolizável aparente da semente de girassol foi bastante alto e não deve ser utilizado como única fonte de alimentação.

Os valores energéticos de alimentos como o milho moído, o farelo de trigo, o germe de trigo, o farelo de soja e a soja micronizada para papagaios foram semelhantes numericamente àqueles encontrados na literatura para galiformes domésticos, principalmente frango de corte.

\section{REFERÊNCIAS BIBLIOGRÁFICAS}

ASSOCIATION OF AMERICAN FEED CONTROL OFFICIALS INCORPORATED. Nutrition expert panel review: new rules for feeding pet birds. Feed Management, Atlanta, v. 49, n. 2, 1998. 
BORGES, F. M. O.; ROSTAGNO, H. S.; RODRIGUEZ, N. M.; SAAD, C. E. P.; TEIXEIRA, E. A.; ARAUJO, V. L. Valores energéticos do grão de trigo e seus subprodutos para frangos de corte. Arquivos Brasileiros de Medicina Veterinária e Zootecnia, Belo Horizonte, v. 55, n. 6, p. 710721, 2003.

CUNNIFF, P. (Ed.). Official methods of analysis of AOAC International. 16. ed. Arlington: AOAC International, 1995. v. 1.

DIERENFELD, E. S.; WENDY, S. G. Manual de nutricion y dietas para animales silvestres en cautiverio: ejemplos para animales de america latina. Bronx: Wildlife Conservation Society, 1996. 110 p.

MARK HAGEN, M. The metabolizable energy of sunflower seed kernelin Goffin's Cockatoo (Cacatua goffini) and a review of the amino acid and fatty acid composition of oil seeds eaten by parrot. Guelph: University of Guelph, 2001. 13 p.
MATTERSON, L. D.; POTTER, L. M.; STUTZ, M. W.; SINGSEN, E. P. The metabolizable energy of feed ingredients for chickens. Storrs: The University of Connecticut, 1965. 11 p. (Research report, 7).

McNAB, J. M.; BLAIR, J. C. Modified assay for true and apparent metabolisable energy based on tube feeding. British Poulry Science, London, v. 29, n. 4, p. 697-707, 1988.

RODRIGUES, P. B.; ROSTAGNO, H. S.; ALBINO, L. F. T. Valores energéticos da soja e subprodutos da soja, determinados com frangos de corte e galos adultos. Revista Brasileira de Zootecnia, Viçosa, v. 31, n. 4, p. 1771-1782, 2002.

WOLF, P. Nutrición de loros. In: CONGRESSO MUNDIAL SOBRE PAPAGAYOS, 5., 2002, Loro Parque. Anais... Loro Parque: Tenerife, 2002. p. 197-205. 